\title{
Erratum to: Phase Stability of Low-Density, Multiprincipal Component Alloys Containing Aluminum, Magnesium, and Lithium
}

\author{
X. YANG, ${ }^{1}$ S.Y. CHEN, ${ }^{1}$ J.D. COTTON, ${ }^{2,3}$ and Y. ZHANG ${ }^{1,4}$ \\ 1.-High-Entropy Theory Center, State Key Laboratory for Advanced Metals and Materials, \\ University of Science and Technology Beijing, Beijing 100083, China. 2.-Metallic Materials \\ Group, The Boeing Company, MC 19-HP, PO Box 3707Seattle, WA 98124-2207, USA. 3.-e-mail: \\ james.d.cotton@boeing.com. 4.—e-mail: drzhangy@ustb.edu.cn
}

\section{ERRATUM TO: JOM, VOL. 66, NO. 10, 2014 \\ DOI 10.1007/S11837-014-1059-Z}

Due to calculation error, Table II, Table V, Fig. 5, and Fig. 6 in the paper were not presented correctly. The updated versions of Table II, Table V, Fig. 5, and Fig. 6 are shown below. 
Table II. Theoretical $\left(\rho_{\text {theor }}\right)$ and measured densities $\left(\rho_{\text {exp }}\right)$, and chemical compositions (at.\%) of phases identified in the microstructures of AlLiMgZnSn, AlLi $\mathbf{A . 5}_{\mathbf{M g Z n}} \mathbf{M n}_{\mathbf{0 . 5}} \mathbf{S n}_{0.2}, \mathbf{A l L i}_{0.5} \mathbf{M g Z n}_{0.5} \mathbf{C u}_{0.2}$, $\mathrm{AlLi}_{0.5} \mathrm{MgCu}_{0.5} \mathrm{Sn}_{0.2}, \mathrm{Al}_{80} \mathrm{Li}_{5} \mathrm{Mg}_{5} \mathrm{Zn}_{5} \mathrm{Sn}_{5}$, and $\mathrm{Al}_{80} \mathrm{Li}_{5} \mathrm{Mg}_{5} \mathrm{Zn}_{5} \mathrm{Cu}_{5}$ alloys

\begin{tabular}{|c|c|c|c|c|c|c|c|c|}
\hline \multirow[b]{2}{*}{ Alloys } & \multirow[b]{2}{*}{$\rho_{\text {theor }}\left(\mathrm{g} / \mathrm{cm}^{3}\right)$} & \multirow[b]{2}{*}{$\rho_{\exp }\left(\mathrm{g} / \mathbf{c m}^{3}\right)$} & \multirow[b]{2}{*}{ Regions } & \multicolumn{5}{|c|}{ Measured chemical composition (at.\%) } \\
\hline & & & & Al & Mg & $\mathbf{Z n}$ & Sn & $\mathbf{C u}$ \\
\hline \multirow[t]{4}{*}{ AlLiMgZnSn } & 3.88 & 4.23 & $\mathrm{~A}$ & 0 & 62.8 & 1.79 & 35.41 & - \\
\hline & & & $\mathrm{B}$ & 54.65 & 0 & 44.59 & 0.76 & - \\
\hline & & & $\mathrm{C}$ & 89.44 & 0 & 10.56 & 0 & - \\
\hline & & & $\mathrm{D}$ & 0 & 0 & 11.31 & 88.69 & - \\
\hline \multirow{3}{*}{$\mathrm{AlLi}_{0.5} \mathrm{MgZn}_{0.5} \mathrm{Sn}_{0.2}$} & 2.90 & 3.22 & $\mathrm{~A}$ & 36.25 & 35.51 & 28.23 & 0 & - \\
\hline & & & $\mathrm{B}$ & 0 & 58.93 & 3.02 & 38.04 & - \\
\hline & & & $\mathrm{C}$ & 83.45 & 12.75 & 3.79 & 0 & - \\
\hline \multirow[t]{3}{*}{$\mathrm{AlLi}_{0.5} \mathrm{MgZn}_{0.5} \mathrm{Cu}_{0.2}$} & 2.75 & 3.73 & $\mathrm{~A}$ & 39.26 & 37.79 & 16.34 & - & 6.61 \\
\hline & & & $\mathrm{B}$ & 32.87 & 32.46 & 15.70 & - & 18.97 \\
\hline & & & $\mathrm{C}$ & 70.36 & 20.12 & 8.77 & - & 0.74 \\
\hline \multirow[t]{3}{*}{$\mathrm{AlLi}_{0.5} \mathrm{MgCu}_{0.5} \mathrm{Sn}_{0.2}$} & 2.96 & 3.69 & $\mathrm{~A}$ & 49.05 & 34.06 & - & 0 & 16.88 \\
\hline & & & $\mathrm{B}$ & 36.76 & 35.09 & - & 0 & 28.15 \\
\hline & & & $\mathrm{C}$ & 4.30 & 22.12 & - & 73.58 & 0 \\
\hline \multirow[t]{2}{*}{$\mathrm{Al}_{80} \mathrm{Li}_{5} \mathrm{Mg}_{5} \mathrm{Zn}_{5} \mathrm{Sn}_{5}$} & 3.05 & 3.05 & A & 87.83 & 1.47 & 3.89 & 6.81 & - \\
\hline & & & B & 8.20 & 2.47 & 3.95 & 85.38 & - \\
\hline \multirow[t]{2}{*}{$\mathrm{Al}_{80} \mathrm{Li}_{5} \mathrm{Mg}_{5} \mathrm{Zn}_{5} \mathrm{Cu}_{5}$} & 2.91 & 3.08 & A & 94.54 & 1.88 & 2.38 & - & 1.20 \\
\hline & & & B & 72.23 & 0 & 1.87 & - & 25.91 \\
\hline
\end{tabular}

Table V. Complete list of investigated alloy compositions, and calculated values for $\Omega, \delta, \Delta \chi$ and VEC

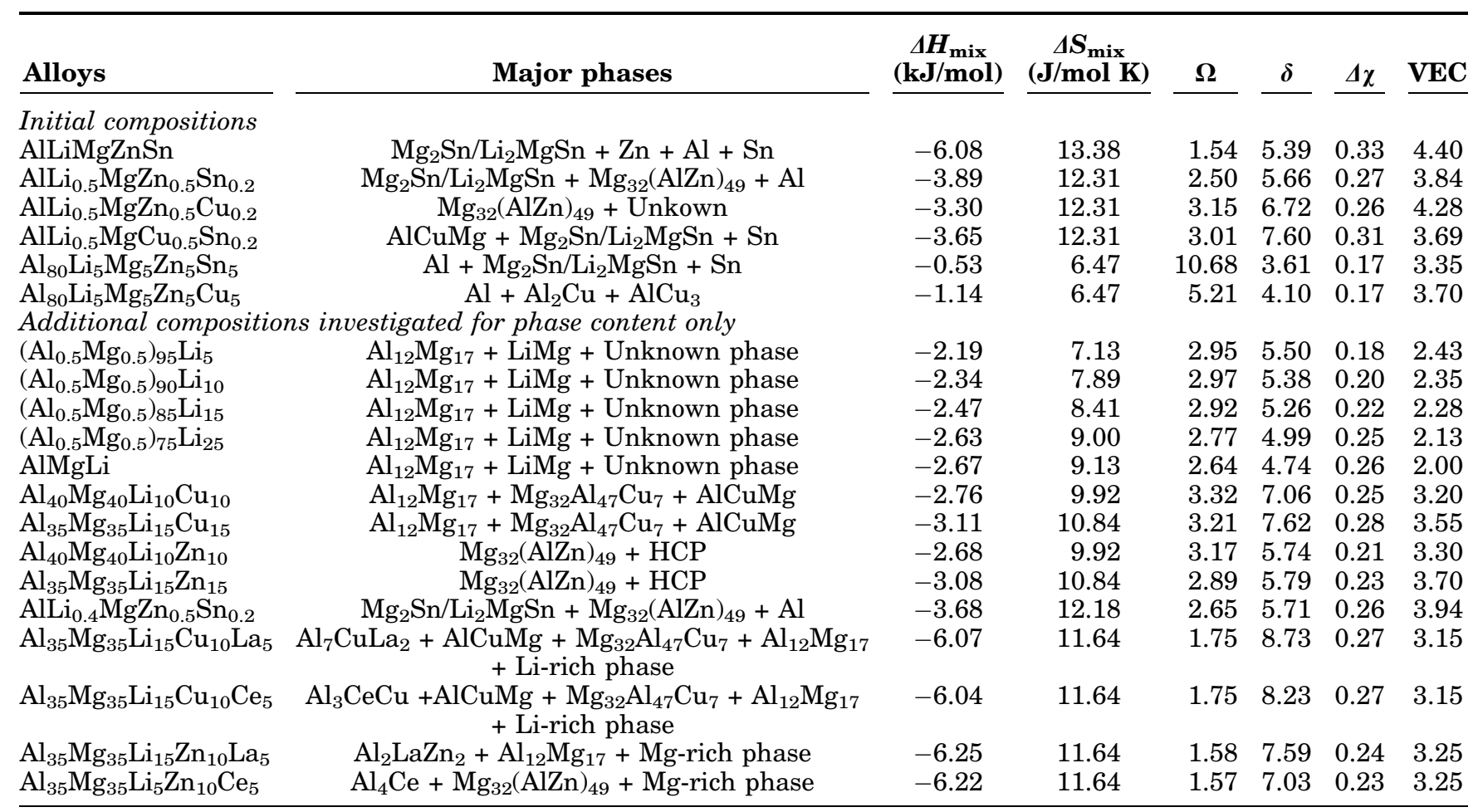



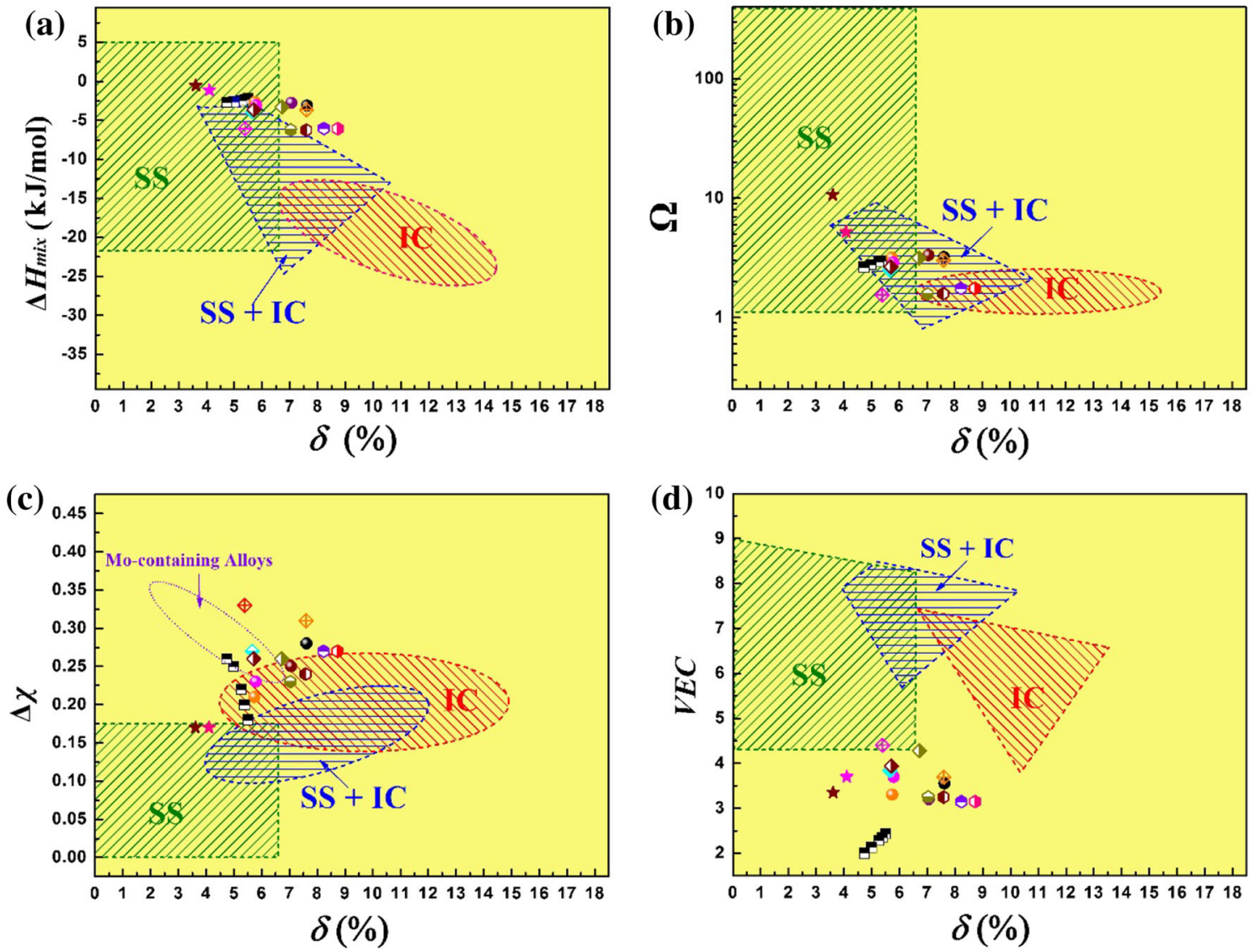

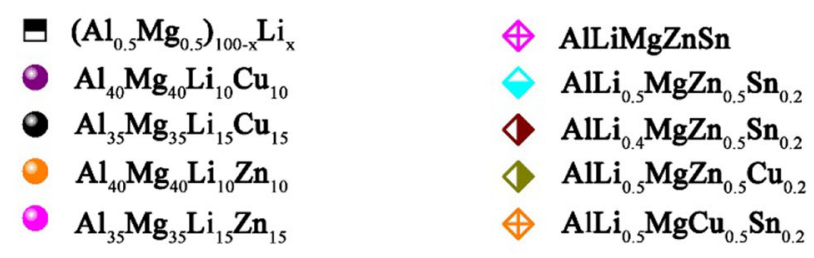

$$
\begin{array}{ll}
\star \mathrm{Al}_{80} \mathrm{Li}_{5} \mathrm{Mg}_{5} \mathrm{Zn}_{5} \mathrm{Sn}_{5} \\
\star \mathrm{Al}_{80} \mathrm{Li}_{5} \mathrm{Mg}_{5} \mathrm{Zn}_{5} \mathrm{Cu}_{5} \\
\text { † } \mathrm{Al}_{35} \mathrm{Mg}_{35} \mathrm{Li}_{15} \mathrm{Cu}_{10} \mathrm{La}_{5} \\
& \mathrm{Al}_{35} \mathrm{Mg}_{35} \mathrm{Li}_{15} \mathrm{Cu}_{10} \mathrm{Ce}_{5} \\
& \mathrm{Al}_{35} \mathrm{Mg}_{35} \mathrm{Li}_{15} \mathrm{Zn}_{10} \mathrm{La}_{5} \\
& \mathrm{Al}_{35} \mathrm{Mg}_{35} \mathrm{Li}_{15} \mathrm{Zn}_{10} \mathrm{Ce}_{5}
\end{array}
$$

Fig. 5. Phase constituent prediction maps: (a) $\delta-\Delta H_{\text {mix }}$; (b) $\delta-\Omega$; (c) $\delta-\Delta \chi$; and (d) $\delta-$ VEC plots for multiprincipal component alloys in this work overlaid on cross-hatched regions developed in previous HEA investigations. (For $\left(\mathrm{Al}_{0.5} \mathrm{Mg}_{0.5}\right)_{100-x} \mathrm{Li} x, x=5,10,15,25$ and 33.33 ). 


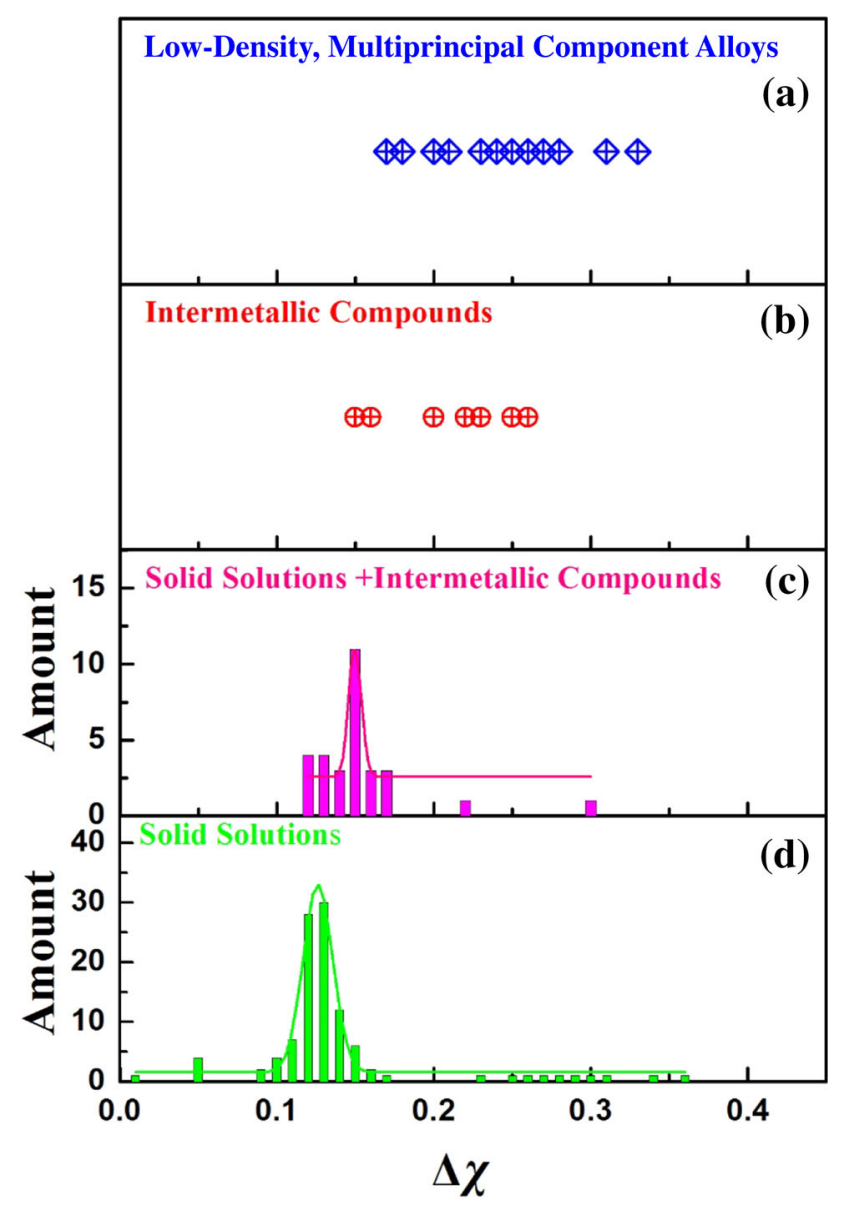

Fig. 6. Values and frequency distributions (c and d) of electronegativity difference $(\Delta \chi)$ for: (a) low-density, multiprincipal component alloys (this work); (b) intermetallic compounds; (c) both solid solutions and intermetallic compounds; and (d) solid solution phases. 